\title{
Performance of the Redfield Ratio and a Family of Nutrient Limitation Indicators as Thresholds for Phytoplankton $\mathbf{N}$ vs. P Limitation
}

\author{
Robert Ptacnik, ${ }^{1,2 *}$ Tom Andersen, ${ }^{3}$ and Timo Tamminen ${ }^{4}$
}

\begin{abstract}
${ }^{1}$ Norwegian Institute for Water Research (NIVA), Gaustadalléen 21, 0349 Oslo, Norway; ${ }^{2}$ Institute for Chemistry and Biology of the Marine Environment (ICBM), Carl von Ossietzky University of Oldenburg, Schleusenstrasse 1, 26384 Wilhelmshaven, Germany; ${ }^{3}$ Department of Biology, University of Oslo, P.O. Box 1066, Blindern 0316, Oslo, Norway; ${ }^{4}$ Marine Research Centre, Finnish Environment Institute (SYKE), P.O. Box 140, 00251 Helsinki, Finland
\end{abstract}

\begin{abstract}
We aim to define the best nutrient limitation indicator predicting phytoplankton biomass increase as a result of nutrient enrichment $(\mathrm{N}, \mathrm{P}$, or both). We compare the abilities of different indicators, based on chemical measurements of nitrogen $(\mathrm{N})$ and phosphorus $(\mathrm{P})$ fractions in the initial plankton community, to predict the limiting factor for phytoplankton growth as inferred independently from short-term laboratory experiments on the same natural communities in a data set from NE Baltic Sea (Tamminen and Andersen, Mar Ecol Prog Ser 340:121-138, 2007). The best indicators had a true positive rate of about $80 \%$ for predicting both $\mathrm{N}$ and $\mathrm{P}$ limitation, but with a higher false positive rate for $\mathrm{N}$ than for P limitation (25 vs. 5\%). Estimated threshold ratios for total nutrients (TN:TP) were substantially higher than the Redfield ratio, reflecting the relatively high amounts of biologically less available dissolved organic $\mathrm{N}$ in the study area. The best overall performing indicator, DIN:TP, had
\end{abstract}

Received 23 September 2009; accepted 27 August 2010; published online 28 October 2010

Electronic supplementary material: The online version of this article (doi:10.1007/s10021-010-9380-z) contains supplementary material, which is available to authorized users.

Author Contributions: RP, TA, TT-all designed study, analyzed data, and wrote the paper

*Corresponding author; e-mail: ptacnik@icbm.de chlorophyll-response based threshold ratios far below Redfield, with $\mathrm{N}$ limitation below 2:1 and $\mathrm{P}$ limitation above 5:1 (by atoms). On the contrary, particulate $\mathrm{N}: \mathrm{P}$ ratio was the overall worst predictor for $\mathrm{N}$ or $\mathrm{P}$ limitation, with values clustering around the Redfield N:P ratio (16:1, by atoms) independent of the limiting factor. Estimated threshold ratios based on inorganic nutrients (DIN:DIP) and so-called biologically available nutrients $(\mathrm{BAN}: \mathrm{BAP}=(\mathrm{PON}+\mathrm{DIN}):(\mathrm{POP}+\mathrm{DIP}))$ were also generally clearly above 16:1, indicating that the Redfield ratio rather reflects the transition from $\mathrm{N}$ limitation to combined $\mathrm{N}+\mathrm{P}$ limitation, than to single limitation by P. Coastal systems are complex systems with regard to nutrient dynamics, historically considered to represent the transition from P-limited freshwater to $\mathrm{N}$-limited marine systems. Our analysis shows that rather simple ratios reflect phytoplankton requirement for nutrients. Based on the high prediction performance, analytical considerations, and general data availability, the DIN:TP ratio appears to be the best indicator for inferring in situ $\mathrm{N}$ vs. P limitation of phytoplankton from chemical monitoring data.

Key words: eutrophication; coastal ecosystems; Baltic sea; stoichiometry; phytoplankton; Redfield ratio; indicators. 


\section{INTRODUCTION}

The Redfield ratio, describing average composition of phytoplankton biomass (Redfield 1934, 1958), is the most broadly applied stoichiometric reference for nutrient limitation of planktonic production. This indicator is recurrently applied from globalscale oceanic biogeochemistry to managementoriented load-response models, and to algal physiology. Redfield originally derived the statistical ratios of key biogenic elements from data for the oceans as a whole', developing a concept of global biogeochemistry based on reciprocal interactions of planktonic biota and their environment over geological time scales.

Redfield emphasized from the beginning that in coastal waters, the ratios of the elements can depart widely from the averages due to proximity to terrestrial nutrient sources, and that the composition of different planktonic species differ. Despite these statements, especially his average N:P (molar) ratio of 16 has been adopted almost as a law of nature, benchmarking a universal nutrient limitation threshold, and has been extended far beyond the original domain of its derivation, up to headwater lakes and algal monocultures in test tubes.

Several recent examinations of the metabolic basis of phytoplankton nutrient limitation and oceanic biogeochemistry (for example, Falkowski 2000; Geider and LaRoche 2002; Arrigo 2005) have deepened our understanding of both nutritional demands of phytoplankton, and of several 'non-Redfield' processes of aquatic ecosystems. There is an acute need, however, for critically analyzed $\mathrm{N}$ vs. $\mathrm{P}$ limitation thresholds in indicators that could be applied to monitoring, modeling, and management of coastal ecosystems (Howarth and Marino 2006).

Degradation of aquatic systems by eutrophication is a global problem calling for well-planned and efficient management strategies. Eutrophication generally refers to increased aquatic production due to increased availability of nutrients, with an array of cascading consequences within the system, such as biomass accumulation, species replacement, and oxygen deficits. Proper identification of the nutrients limiting phytoplankton growth is thus a necessary prerequisite for successful eutrophication reduction at impacted sites where future changes in nutrient discharges may alter the prevailing limitation patterns (Cloern 2001; Ptacnik and others 2005).

Historically, marine and terrestrial systems have been considered primarily $\mathrm{N}$-limited (Howarth and Marino 2006; Vitousek and Howarth 1991), whereas predominant $\mathrm{P}$ limitation has been the paradigm for lakes (Schindler 1977). Coastal systems represent transitional zones between terrestrial, freshwater, and marine nutrient cycles, appearing thus specifically to call for reliable $\mathrm{N}$ vs. $\mathrm{P}$ limitation indicators. However, a recent metaanalysis questioned any basic dichotomy in nutrient limitation between different habitats, by finding surprising similarity across major ecosystem types in terms of $\mathrm{N}$ and $\mathrm{P}$ limitation (Elser and others 2007). Moreover, they found widespread co-limitation by $\mathrm{N}$ and $\mathrm{P}$ in all systems studied, indicating closely balanced $\mathrm{N}$ and $\mathrm{P}$ supplies in most environments. This calls attention to the need for local assessments of limiting nutrients in environmental management, but also sets demands for limitation indicators to be able to distinguish between $\mathrm{N}, \mathrm{P}$, and combined $\mathrm{N}$ and $P$ limitation.

Here we investigate how in situ phytoplankton nutrient limitation is related to different fractions of ambient nutrient concentrations, and aim at developing an optimal predictor for the primary limiting nutrient. Instead of studying the Redfield ratio through statistical analysis of extensive field data (Redfield 1934, 1958) or from the first principles of phytoplankton metabolism (Falkowski 2000; Geider and LaRoche 2002), we adopt an approach that might be described as heuristic. We base our analysis on a large coastal dataset consisting of a 3-year series of bioassay experiments assessing patterns of nutrient limitation of phytoplankton, based on both chlorophyll $a$ and primary productivity responses in $\mathrm{P}$ - and $\mathrm{N}$-limited sub-basins of the Baltic Sea (Tamminen and Andersen 2007; Andersen and others 2007).

We take these experimentally verified phytoplankton limitation patterns as our starting point, collapse the seven generic limitation categories (Andersen and others 2007) into a univariate limitation indicator, and relate this information on observed phytoplankton limitation to initial nutrient data. We employ hyperbolic tangent functions to derive chemical limitation indicators with constant and non-constant (that is, scaling with nutrient concentrations) threshold $\mathrm{N}: \mathrm{P}$ ratios for the transitions between $\mathrm{N}, \mathrm{N}+\mathrm{P}$, and $\mathrm{P}$ limitation. Finally, we analyze the performance of the indicators quantitatively with multiple criteria (coefficient of determination and Akaike Information Criterion regression statistics, as well as Receiver Operating Characteristics plots). 


\section{Materials and Methods}

\section{Experimental Set-Up and Analyses}

A detailed description of the study area and bioassay experiments is given in Tamminen and Andersen (2007); therefore only a brief sketch is provided here. During the growth seasons of 1992-1994, 3-day time-series bioassay experiments were carried out at regular time intervals over the season at six monitoring stations along the $1000 \mathrm{~km}$ of Finnish coastline, spanning the large-scale Baltic Sea gradients in salinity and eutrophication, and also a continuum from $\mathrm{P}$ to $\mathrm{N}$ limitation (Tamminen and Andersen 2007). A correlation analysis showed overall low association among major environmental variables (see Supplementary material). In particular, correlations among salinity and major nutrient fractions were negligible. Hence it seems unlikely that potential relationships between limitation patterns and nutrient concentrations will be confounded by an underlying salinity gradient.

Seven to 12 experiments were done per station and year, resulting in a total of 170 experiments. Each experiment was started by taking an initial sample from a monitoring station. An array of physical and chemical parameters was measured on this non-manipulated initial sample using standard methods. The following initial analyses were used for constructing the limitation indicators of this study: dissolved inorganic nitrogen (DIN) as the sum of ammonium, nitrate and nitrite; dissolved inorganic phosphate (DIP); total N and P (TN, TP); all measured in duplicate with wet chemistry and photometric detection according to Grasshoff and others (1983). In addition, particulate P (POP) was measured after Solorzano and Sharp (1980), and particulate $\mathrm{N}(\mathrm{PON})$ with a mass spectrometer (Europa Scientific), both after filtering duplicate samples onto glass-fiber filters (Whatman GF/F; acid-washed and precombusted at $500^{\circ} \mathrm{C}$ ).

Each experiment consisted of eight subsamples $(6 \mathrm{l})$ from the initial sample, amended with phosphorus $\left(0.65 \mu \mathrm{mol} \mathrm{l}^{-1}\right)$ and ammonium $(2.86$ $\mu \mathrm{mol} \mathrm{l}^{-1}$ ) additions according to a $2 \times 2$ factorial design with replicates. Treatments were incubated for $72 \mathrm{~h}$ at in situ temperature and a photoperiod corresponding to the time and location of the sampling (light intensity: $100 \mu \mathrm{mol}$ quanta $\mathrm{m}^{-2} \mathrm{~s}^{-1}$ ). All experimental units were sampled for chlorophyll $a$ on days $1-3$, and ${ }^{14} \mathrm{C}$-uptake was measured on days 1 and 2. Parameters were measured in duplicate, giving a total of 50 chlorophyll $a$ samples and $34{ }^{14} \mathrm{C}$-samples from each experiment (including the initial measurements). Methods for the experiment response analyses are detailed in Tamminen and Andersen (2007).

\section{Statistical Analysis of the Bioassay Experiments}

The most probable nutrient limitation in a given experiment was inferred by a statistical model selection procedure contrasting 21 different candidate models representing biologically consistent response patterns. A resampling approach based on the lowest replication level was used for estimating probabilities for different limitation categories in each experiment. These probabilities also represent uncertainties in the limitation class assignments; an experiment with low classification uncertainty would have nearly all probability concentrated on a single category, while an ambiguous experiment would have the probabilities spread out between many limitation classes (Andersen and others 2007).

\section{Creating a Univariate Limitation Indicator}

Andersen and others (2007) found that factorial experiments with two limiting nutrients can be described by seven generic limitation patterns, which allow distinguishing between exclusive, primary, and combined limitation. Exclusive limitation is when only one of the nutrients leads to a growth response and the other nutrient is nonlimiting throughout the experiment, while primary limitation is when an initially non-limiting nutrient becomes limiting after the primary limiting nutrient is added. For our analysis, we first simplified this scheme by merging the exclusive and primary limitation classes for a particular nutrient, resulting in three basic limitation categories corresponding to $\mathrm{N}, \mathrm{P}$, or combined $\mathrm{N}$ and $\mathrm{P}$ limitation. By subtracting the probability of $\mathrm{N}$-limitation from the corresponding probability of P-limitation we got a one-dimensional indicator $(L)$ on the interval from -1 to +1 , where the endpoints represent pure $\mathrm{N}$ - and P-limitation, respectively, and the midpoint represents combined or no limitation. To illustrate the general principle, we have reproduced the time course of one particular experiment in Figure 1A (from the Helsinki district (Gulf of Finland) June, 1994). Since there were significantly different effects of all combinations of $\mathrm{N}$ and $\mathrm{P}$ addition, this experiment would be classified as combined $\mathrm{N}$ and $\mathrm{P}$ limitation. Figure 1B shows that this conclusion is very sensitive to small variations in the input data: over $80 \%$ of the bootstrap samples of the original gave a simpler model with only effects of $\mathrm{N}$ 

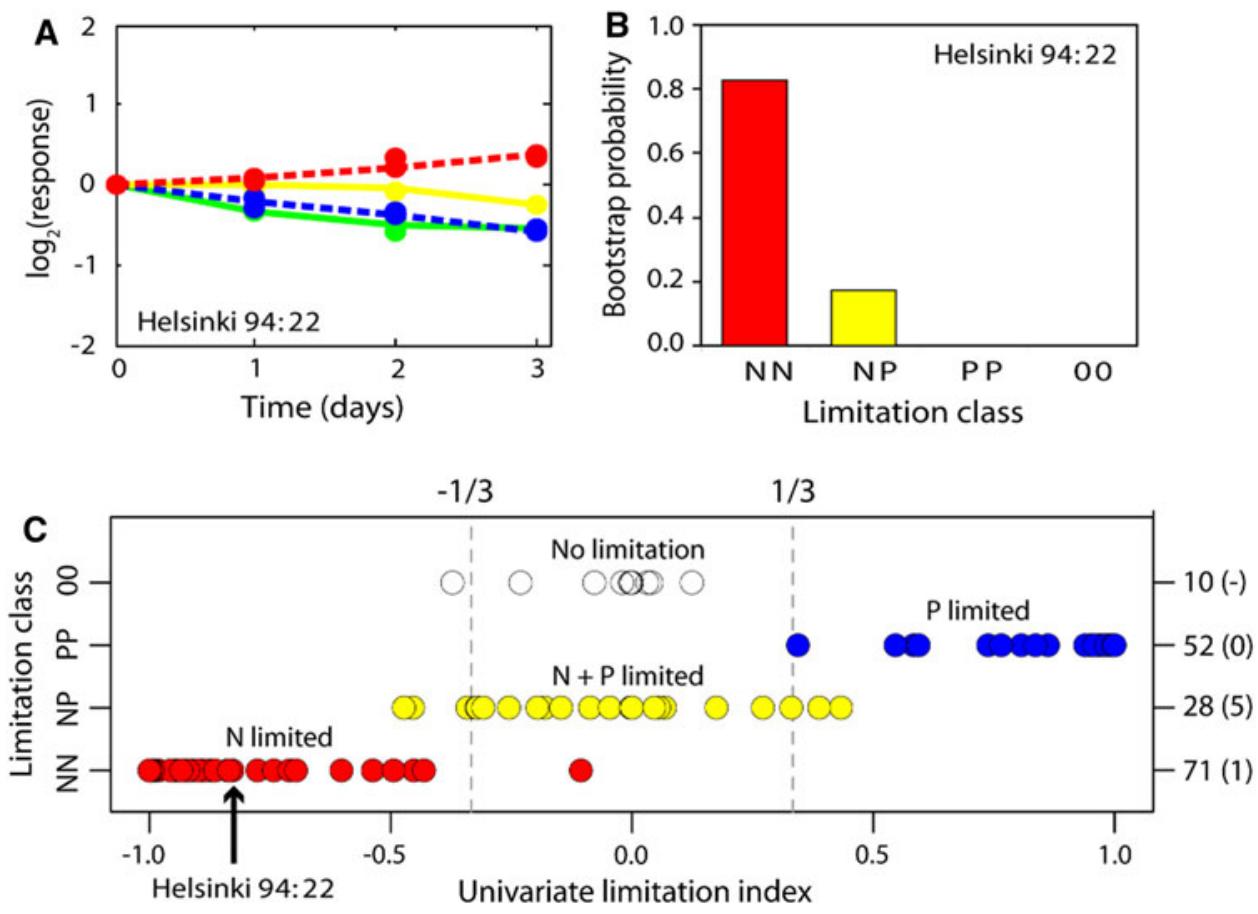

Figure 1. Linking nutrient limitation classes to the univariate limitation index: A Example of a time course for a single limitation experiment (Helsiki district, June 1994) with measurements and model predictions according to Andersen and others (2007) in blue (no addition), green (P only), yellow ( $\mathrm{N}$ only), and red $(\mathrm{N}+\mathrm{P})$. B The corresponding limitation class probabilities from 100 bootstrap samples on the bottle-level replicates of the experiment in $\mathbf{A}$, classified into $\mathrm{N}$, $\mathrm{P}$ or, combined limitation (NN, PP, or NP), or no limitation (00). C Univariate limitation index ( $x$-axis), transforming N, P, and combined limitation onto a one-dimensional scale for all experiments. The numbers of experiments with complete correspondence between discrete limitation categories and the univariate index are given by the right $y$-axis, with numbers of conflicting classes in parenthesis. Black arrow indicates the position of the example data set in $\mathbf{A}$ and $\mathbf{B}$.

addition while less than $20 \%$ maintained the original classification, giving a strongly negative score on the univariate limitation index (Figure 2C, arrow). As this univariate limitation indicator cannot distinguish experiments with no limitation from those with combined limitation, we chose to exclude the experiments that were classified as non-limited by Tamminen and Andersen (2007) from further analysis. This reduced the data set by 10 experiments for chlorophyll $a$ response and 12 experiments for ${ }^{14} \mathrm{C}$ response. In addition, a small number of experiments did not produce a full set of nutrient data for indicator calculations, leaving 145 experiments for the current analysis for both response variables.

\section{Hyperbolic Tangent Regressions}

The limitation indicator $(L)$ was fitted to hyperbolic tangent functions of the form

$$
\begin{gathered}
L=\tanh (a+b \log (N / P)) \\
L=\tanh (a+b \log (N)+c \log (P))
\end{gathered}
$$

where $a, b$, and $c$ are constants, and $N$ and $P$ represent various fractions of nitrogen and phosphorus, respectively. Both models have level curves for constant limitation indicator values that are straight lines in the plane spanned by the logtransformed predictors (for example, $\log (\mathrm{TN})$ and $\log (\mathrm{TP}))$. The main difference is that model (1) will have isolines with unit slope, while the isoline slopes of model (2) will be different from 1 unless $c=-b$ (in which case models (1) and (2) are identical). Thus, model (1) will have a constant threshold N:P ratio for the transition between N and P limitation, while in model (2) the threshold ratio is non-constant and dependent on absolute nutrient levels. The hyperbolic tangent fits nicely with the span of the chosen limitation indicator, but is otherwise just a translation and rescaling of the logistic response function commonly used in toxicology and risk analysis. Regression fitting was done using the nonlinear least squares function (nls) of $\mathrm{R}$ version 2.4 (R Development Core team 2005). As starting values for fitting equation (1) we used $a=-m / s$ and $b=1 / S$, where $m$ and $S$ are the mean and 

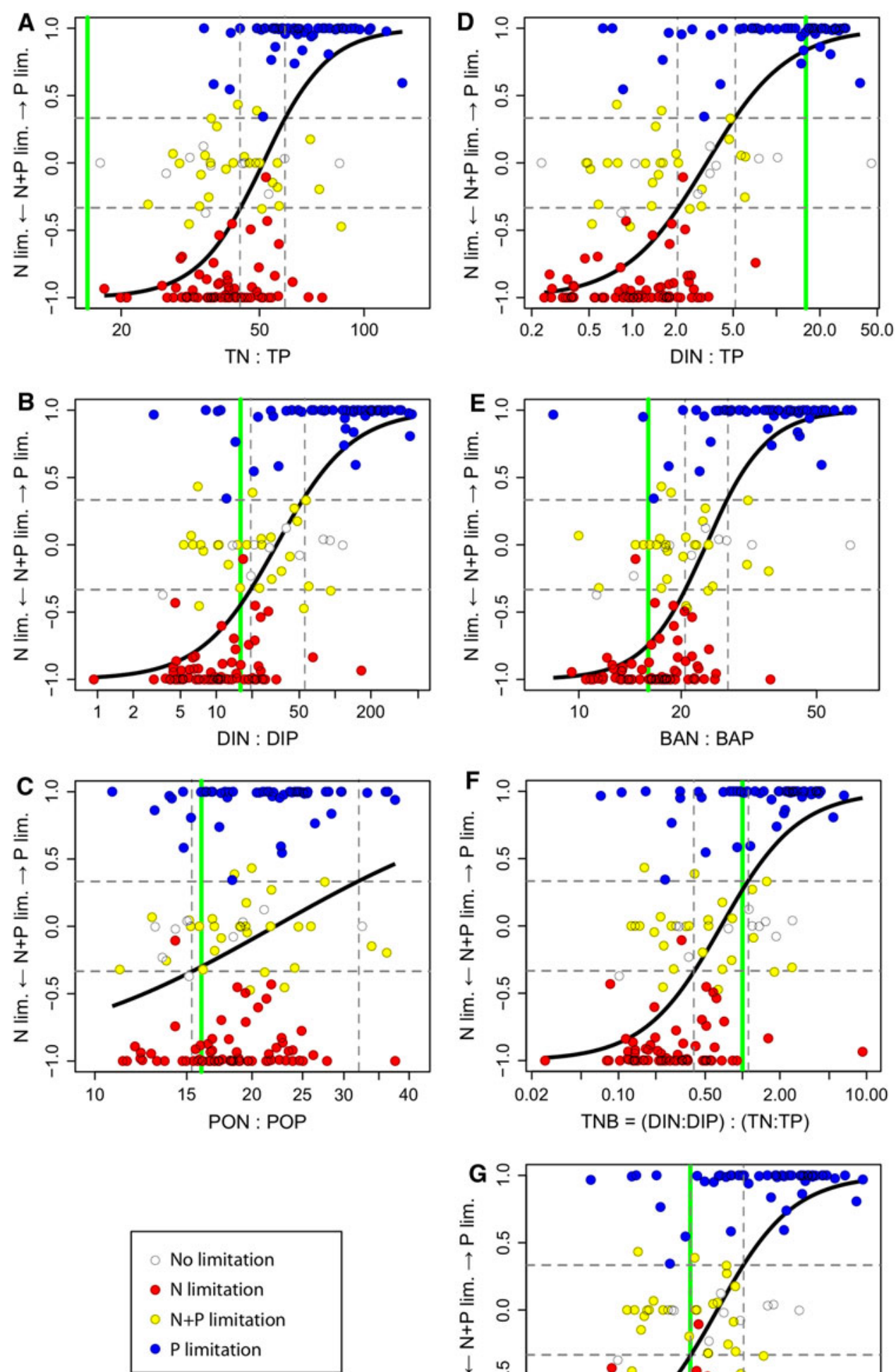
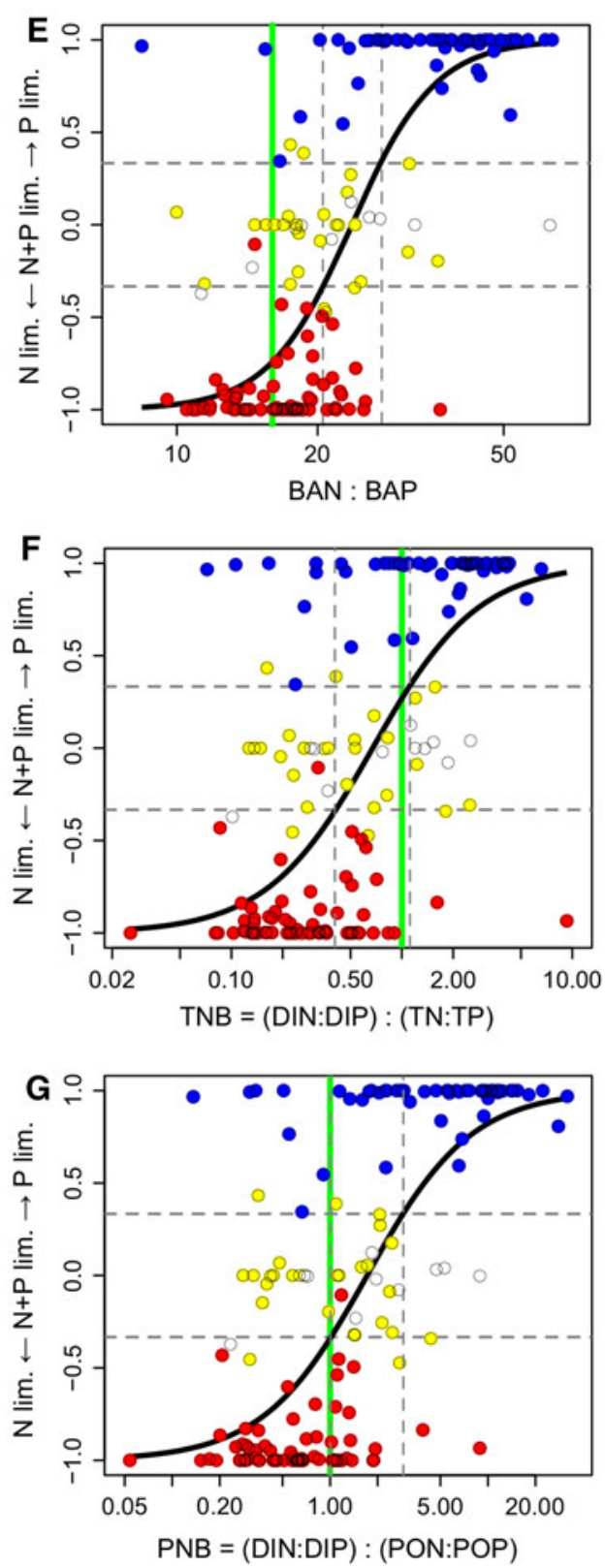

Figure 2. Scatter-plots presenting the 1D models for standard and composite indicators (chlorophyll a responses; see "Materials and Methods" section for description). Each dot represents the outcome of one experiment. The color code gives the most probable limitation as revealed by bootstrap analysis of the modeled chlorophyll $a$ responses (Tamminen and Andersen 2007). The solid black line represents the prediction of combined limitation (midpoint of the univariate index) by the corresponding $1 \mathrm{D}$ regression model.

Horizontal dashed lines separate the experimentally verified transitions between limitation categories ( $\mathrm{N}$, combined, P) according to the univariate limitation index. Vertical dashed lines denote corresponding transitions predicted by the ID model for the indicator. The green line indicates the Redfield ratio (16 mol: $\mathrm{mol}$ ) in $\mathbf{A}-\mathbf{E}$ and the $1: 1$ ratio in $\mathbf{F}-\mathbf{G}$. 
standard deviation of $\log (N / P)$. The fitted parameter values were then used as starting values for fitting equation (2), with the additional parameter $c$ set equal to $-b$.

A variety of physical parameters and nutrient fractions was originally analyzed from the initial water samples, covering a normal monitoring program suite of measurements, but also augmented with organic nutrient fractions (dissolved and particulate). Preliminary data analysis indicated little or no relationship of nutrient limitation with physical parameters and dissolved organic nutrients. These factors were therefore not considered for the development of indicators.

\section{Potential Limitation Indicators}

The following N:P ratios were tested as predictors for the observed nutrient limitation:

- three 'standard' $\mathrm{N}: \mathrm{P}$ ratios between matching nutrient fractions: dissolved inorganic (DIN:DIP); total (TN:TP); and particulate organic fractions (PON:POP);

- two 'composite' N:P ratios: DIN:TP ratio after Morris and Lewis (1988); and the ratio of bioavailable nutrients (BAN:BAP; Reynolds and Davis 2001), representing the ratio between the sums of biologically available $\mathrm{N}$ and $\mathrm{P}$ ((DIN + PON):(DIP + POP));

- two 'nutrient balance ratios' (Tamminen 1982), where the supply ratio of dissolved inorganic $\mathrm{N}$ and P (DIN:DIP) is compared to the demand ratio of either total (TN:TP), or particulate organic nutrients (PON:POP); TNB = (DIN:DIP):(TN:TP); and $\mathrm{PNB}=(\mathrm{DIN}: \mathrm{DIP}):(\mathrm{PON}: \mathrm{POP})$.

The two last-mentioned indicators (TNB and PNB) are expected to have a critical ratio around unity (that is, the $\mathrm{N}: \mathrm{P}$ ratio of supplies matches the demand), while the other predictor candidates would be expected to have critical levels closer to the Redfield ratio ( $\mathrm{N}: \mathrm{P}=16$, by atoms). All predictors were analyzed in their one- and two-dimensional form (see equations (1) and (2)).

\section{Goodness of predictors}

Relative performance of the limitation predictors was compared by regression statistics of the 2 models (equations (1) and (2)) for each predictor. The criteria used were the Akaike Information Criterion (AIC; Johnson and Omland 2004), which depends on both the goodness of fit and the complexity of the model, and coefficient of determination $\left(R^{2}\right)$ describing the fraction of variance explained by the model. In addition to this, we used Receiver Operating Characteristics (ROC) plots for visualizing the performance of the limitation classifiers (Fawcett 2006). These plots, commonly used in medical decision making but recently also in machine learning and data mining research, compare the match between classifier predictions and known classes of a data set, after composing two-by-two confusion matrices (contingency tables; see Table 2 in Supplementary material) containing perfect matches (true positive or negative) and false classifications (false positive or negative). The graph plots true positive rate (or hit rate) against false positive rate (or false alarm rate) of the classifiers against each other, thus depicting relative tradeoffs between benefits (true positives) and costs (false positives).

\section{Threshold N:P Ratios}

The ID models (equation (1)) have concentrationindependent threshold $\mathrm{N}: \mathrm{P}$ ratios for the transitions from $\mathrm{N}$ to $\mathrm{N}+\mathrm{P}$ limitation at $L=-1 / 3$, and from $\mathrm{N}+\mathrm{P}$ limitation to $\mathrm{P}$ limitation at $L=1 / 3$. If $a$ and $b$ are fitted parameters to equation (1), then the threshold N:P ratios for a given indicator can be calculated as

$$
(N / P)_{\text {Threshold }}=\exp \left(\left(\tanh ^{-1}( \pm 1 / 3)-a\right) / b\right)
$$

We used a bootstrapping approach to estimate $95 \%$ confidence limits for the threshold N:P ratios by resampling 1000 times with replacement from the original data, refitting the model parameters $a$ and $b$, and calculating a bootstrap distribution of threshold N:P ratios by equation (3).

\section{RESUltS}

The univariate limitation indicator created from the seven generic limitation patterns of Andersen and others (2007) preserved well the information content of the original limitation classes (Figure 1), in spite of the information lost by compression into one dimension. Classifications based on dividing the range of the univariate limitation index into three equal segments recovered the original limitation classes in $82 \%$ (combined limitation), 98\% $(\mathrm{N})$, or $100 \%(\mathrm{P})$ of the cases (Figure 1). These numbers correspond to the true positive rates (Fawcett 2006) of classifiers based on the univariate limitation index, which can also be taken as an upper performance limit for alternative indicators based on nutrient concentrations at the start of the experiment. 
Table 1. Comparison of Various Indicator Models Predicting the Prevailing Limiting Nutrient Described by the Univariate Limitation Index (Range -1 to +1 , See "Materials and Methods" Section, Figure 1), Summarizing Modeled Phytoplankton Responses in Experiments of Tamminen and Andersen (2007)

\begin{tabular}{llllllll}
\hline & TN:TP & DIN:DIP & PON:POP & DIN:TP & BAN:BAP & TNB & PNB \\
\hline Chl-1D & & & & & & & \\
$a$ & $-9.0 \pm 1.4$ & $-2.3 \pm 0.3$ & $-3.0 \pm 0.9$ & $-0.9 \pm 0.1$ & $-7.6 \pm 1.1$ & $0.27 \pm 0.10$ & $-0.34 \pm 0.08$ \\
$b$ & $2.3 \pm 0.4$ & $0.7 \pm 0.1$ & $1.0 \pm 0.3$ & $0.8 \pm 0.1$ & $2.4 \pm 0.4$ & $0.69 \pm 0.11$ & $0.66 \pm 0.10$ \\
$\mathrm{~N} \rightarrow \mathrm{P}$ & $44 \rightarrow 59$ & $19 \rightarrow 53$ & $15 \rightarrow 31$ & $2.0 \rightarrow 5.1$ & $21 \rightarrow 27$ & $0.4 \rightarrow 1.1$ & $1.0 \rightarrow 2.8$ \\
$\mathrm{AIC}\left(R^{2}\right)$ & $285(0.44)$ & $257(0.53)$ & $\underline{356(0.08)}$ & $\mathbf{2 3 5 ( \mathbf { 0 . 6 0 } )}$ & $245(0.57)$ & $287(0.43)$ & $273(0.48)$ \\
$\mathrm{Chl}-2 \mathrm{D}$ & & & & & & \\
$a$ & $-10.3 \pm 2.2$ & $-1.7 \pm 0.3$ & $-3.2 \pm 0.9$ & $-1.3 \pm 0.2$ & $-7.3 \pm 1.1$ & $-6.9 \pm 1.1$ & $-3.9 \pm 0.9$ \\
$b$ & $2.7 \pm 0.6$ & $0.7 \pm 0.1$ & $0.6 \pm 0.3$ & $0.7 \pm 0.1$ & $2.2 \pm 0.4$ & $0.6 \pm 0.1$ & $0.6 \pm 0.1$ \\
$\mathrm{c}$ & $-2.4 \pm 0.4$ & $-0.5 \pm 0.1$ & $-1.6 \pm 0.4$ & $-1.3 \pm 0.2$ & $-2.5 \pm 0.4$ & $1.3 \pm 0.3$ & $0.6 \pm 0.3$ \\
$\mathrm{AIC}\left(R^{2}\right)$ & $286(0.44)$ & $253(0.55)$ & $\underline{330(0.24)}$ & $\mathbf{2 2 7}(\mathbf{0 . 6 3})$ & $244(0.58)$ & $233(0.61)$ & $255(0.55)$ \\
${ }^{14} \mathrm{C}-1 \mathrm{D}$ & & & & & & & \\
$a$ & $-8.0 \pm 1.4$ & $-1.4 \pm 0.3$ & $-1.7 \pm 0.9$ & $-0.25 \pm 0.08$ & $-4.4 \pm 0.8$ & $0.46 \pm 0.11$ & $0.07 \pm 0.07$ \\
$b$ & $2.1 \pm 0.4$ & $0.5 \pm 0.1$ & $0.6 \pm 0.3$ & $0.50 \pm 0.09$ & $1.5 \pm 0.3$ & $0.44 \pm 0.08$ & $0.46 \pm 0.08$ \\
$\mathrm{~N} \rightarrow \mathrm{P}$ & $37 \rightarrow 52$ & $8 \rightarrow 34$ & $9 \rightarrow 26$ & $0.8 \rightarrow 3.3$ & $16 \rightarrow 25$ & $0.16 \rightarrow 0.77$ & $0.41 \rightarrow 1.83$ \\
$\mathrm{AIC}\left(R^{2}\right)$ & $301(0.38)$ & $302(0.37)$ & $\underline{365(0.04)}$ & $\mathbf{3 0 0}(\mathbf{0 . 3 8})$ & $308(0.35)$ & $326(0.26)$ & $311(0.33)$ \\
${ }^{14} \mathrm{C}-2 \mathrm{D}$ & & & & & & & \\
$a$ & $-10.6 \pm 2.2$ & $-1.3 \pm 0.4$ & $-1.8 \pm 0.9$ & $-0.53 \pm 0.15$ & $-4.5 \pm 0.8$ & $-6.1 \pm 1.2$ & $-2.6 \pm 0.9$ \\
$b$ & $2.9 \pm 0.7$ & $0.52 \pm 0.10$ & $0.47 \pm 0.30$ & $0.41 \pm 0.09$ & $1.4 \pm 0.3$ & $0.4 \pm 0.1$ & $0.48 \pm 0.08$ \\
$\mathrm{c}$ & $-2.2 \pm 0.4$ & $-0.44 \pm 0.12$ & $-0.95 \pm 0.33$ & $-0.90 \pm 0.21$ & $-1.8 \pm 0.3$ & $1.3 \pm 0.3$ & $0.43 \pm 0.29$ \\
$\mathrm{AIC}\left(R^{2}\right)$ & $300(0.39)$ & $304(0.38)$ & $\underline{357(0.10)}$ & $297(0.41)$ & $307(0.36)$ & $\mathbf{2 7 5}(\mathbf{0 . 4 9})$ & $302(0.39)$
\end{tabular}

Regression coefficients (a, b, c; \pm standard errors) are given for the $1 D$ and $2 D$ models (see equations (1) and (2)), as well as the indicator threshold values for transitions between limitation categories ( $N$, combined, $P$ ) predicted by $1 D$ models $(N \rightarrow P$, corresponding to vertical dashed lines in Figures 2 and 4$)$. In addition, Akaike Information Criterion (AIC) value and coefficient of determination $\left(R^{2}\right.$, in brackets) are presented for each model. Goodness of prediction increases with decreasing AIC value, and increasing $R^{2}$. For each model type (Chl-1D, Chl-2D, $\left.{ }^{14} \mathrm{C}-1 \mathrm{D},{ }^{14} \mathrm{C}-2 \mathrm{D}\right)$, the best limitation predictor by these criteria is printed in boldface, the two closest contenders in italics, and the worst underlined.

All the tested indicators yielded highly significant models, with the exception of PON:POP. The twodimensional versions of the predictors gave consistently better results than their one-dimensional counterparts as indicated by both AIC and regression $R^{2}$ (Table 1), indicating that the threshold ratio for a switch from a limitation type to another is not constant, but depends on nutrient concentrations. Although the ranking of the indicators was roughly similar regardless of indicator model type or limitation response variable, all indicators predicted limitation categories based on experimental chlorophyll responses better than those based on responses in ${ }^{14} \mathrm{C}$ primary production (Table 1). Detailed analyses are only presented for chlorophyll-based responses, while results for ${ }^{14} \mathrm{C}$ limitation models are summarized in Table 1 . For the corresponding treatment for ${ }^{14} \mathrm{C}$ limitation responses see Supplementary material.

\section{Standard N:P Indicators (TN:TP, DIN:DIP, PON:POP)}

The traditional applications of the Redfield ratio performed very differently from each other. All
TN:TP data, including the P-limited experiments, significantly exceeded the Redfield ratio of 16, reaching values up to 100 , whereas PON:POP was more constrained, from 10 to 40 (Figure 2A, C). This clearly points out a large excess of dissolved organic $\mathrm{N}$ that is unavailable for the phytoplankton (Supplementary material).

The 1D model for TN:TP showed only moderate predictive power, with values around 50 for shifts in limitation (Table 1; Figure 2A), whereas the corresponding PON:POP model had very low predictive power $\left(R^{2}=0.07\right.$; Table 1$)$, as visualized by the completely overlapping limitation categories over the full range of PON:POP (Figure 2C). The 2D models for these ratios (Figure 3A, C) improved the performance of PON:POP, but it still clearly remained the weakest of all predictors. Prediction power of TN:TP was not enhanced by the concentration-dependent 2D model (Table 1).

DIN:DIP demonstrated the widest range of values, covering more than two orders of magnitude, with shifts from $\mathrm{P}$ to combined to N-limitation far above the Redfield ratio (19 and 53, respectively; Table 1; Figure 2B). The predictive power of DIN:DIP was clearly the best of standard ratios, and it 

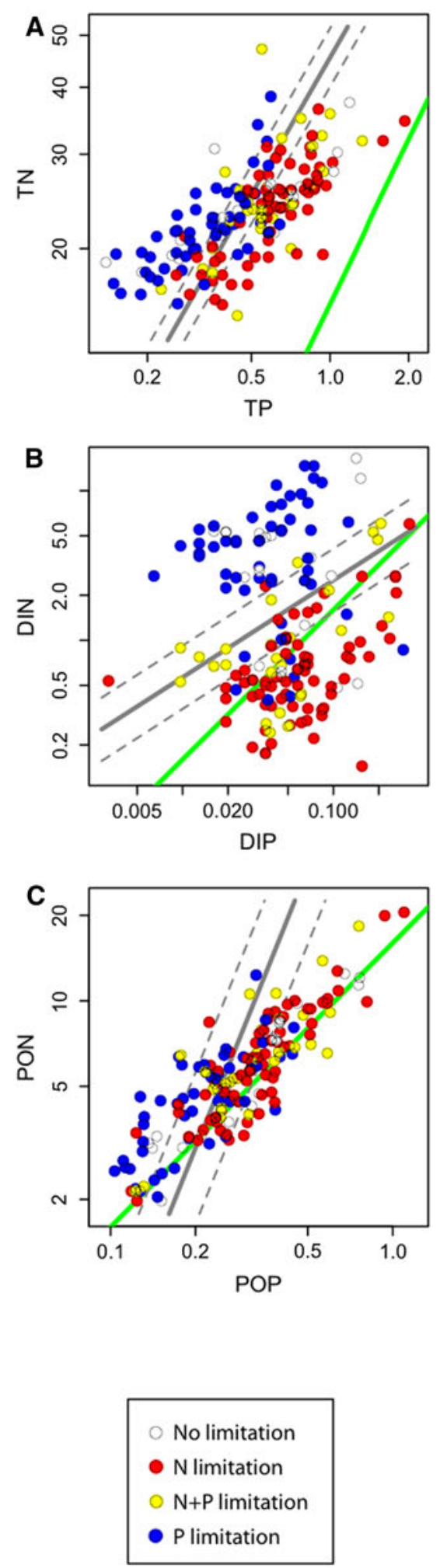
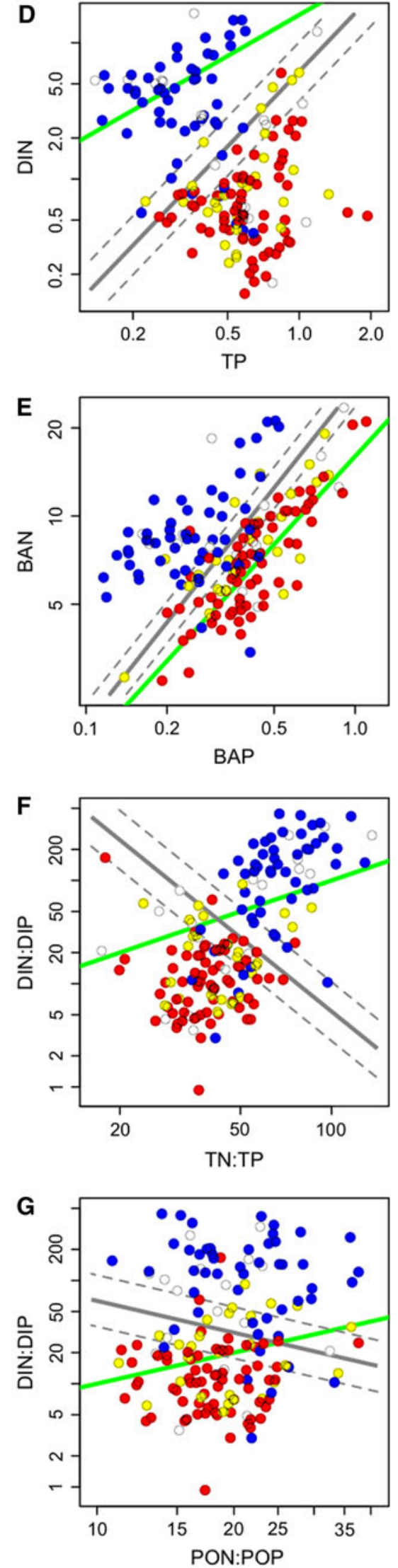

Figure 3. Scatter-plots presenting the 2D models for standard and composite indicators (chlorophyll $a$ responses; see "Materials and Methods" section for description). Each dot represents the outcome of one experiment. The color code gives the most probable limitation as revealed by bootstrap analysis of the modeled chlorophyll $a$ responses (Tamminen and Andersen 2007). The solid gray line represents the prediction of combined limitation (midpoint of the univariate index) by the corresponding $2 \mathrm{D}$ regression model, the dashed lines give the predicted interval of combined limitation (univariate limitation index between $-1 / 3$ and $1 / 3)$. The green line indicates the Redfield ratio ( $16 \mathrm{~mol}: \mathrm{mol}$ ) in $\mathbf{A}-\mathbf{E}$ and the 1:1 ratio in $\mathbf{F}-\mathbf{G}$. 


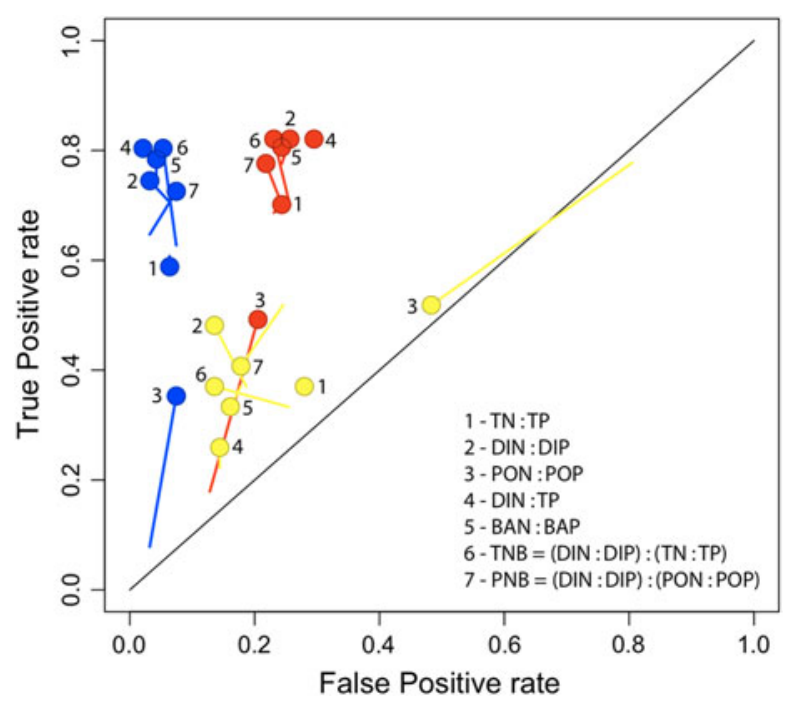

Figure 4. Receiver Operating Characteristics (ROC) plot for visualizing the performance of the limitation indicators coded by numbers and grouped by color codes according to the most probable limitation inferred from bootstrap analysis of the modeled chlorophyll $a$ responses (Tamminen and Andersen 2007). The graph shows true positive rate ( $y$-axis) against false positive rate ( $x$-axis) of each set of indicator predictions, illustrating thus the match and error risk between indicator prediction and known limitation. The pins connect corresponding ID (sharp end) and 2D (ball end) model predictions of each indicator. Upper left corner represents perfect match, dotted diagonal 50:50 hit rate, and lower right corner total failure.

was only slightly enhanced by the $2 \mathrm{D}$ model (Table 1). The 2D model showed, however, that the regression departed from Redfield slope, indicating concentration-dependency towards low inorganic nutrient concentrations (Figure 3B).

\section{Composite N:P Indicators (DIN:TP, BAN:BAP)}

The composite indicators performed the best according to regression statistics, with DIN:TP scoring the highest coefficient of determination of all indicators with the 2D model $\left(R^{2}=0.64\right)$, and only slightly less with the $1 \mathrm{D}$ model (Table 1). BAN:BAP followed closely, with almost identical scores for $1 \mathrm{D}$ and 2D models. The range for limitation shifts in DIN:TP was from 2 to 5 (Table 1; Figure 2D), significantly below the Redfield N:P ratio, whereas the biologically available fractions (BAN:BAP) indicated limitation shifts above 16 (21 and 27; Table 1; Figure 2E). The 2D models showed a slight displacement of BAN:BAP slope compared to Redfield, whereas the DIN:TP slope deviated markedly and exhibited clear concentrationdependency (Figure 3D, E).

\section{Nutrient Balance Ratios (TNB, PNB)}

Apart from PON:POP, the nutrient balance ratios benefited the most from 2D models compared to corresponding 1D ones (Table 1). The shifts between limitation categories in ID models took place near unity (Table 1; Figure 2F, G), but corresponding $2 \mathrm{D}$ models showed that enhancing the predictive capacity of the ratios required clear departure from unity, especially for TNB (Figure 3F, G). The 2D model for this ratio scored very close to the best chlorophyll-based model (DIN:TP) according to regression statistics $\left(R^{2}=\right.$ 0.62 ; Table 1), and was clearly the best of all for ${ }^{14} \mathrm{C}$-based models (Table 1).

\section{ROC Summary}

The Receiver Operating Characteristics plot (Figure 4) summarizes the predictive power of the indicators, demonstrating a clear clustering of prediction confidence by the limitation category $(\mathrm{N}, \mathrm{P}$, or combined). P limitation was generally predicted with high true positive rate (around $80 \%$ ) and low false positive rate $(<10 \%)$, with the exception of the very low performance of PON:POP. N limitation was also generally predicted with a high true positive rate, except for PON:POP, but with clearly increased false positive rate $(20-30 \%)$. Combined limitation prediction was characterized by decreased true positive rate with larger scatter of the indicators $(25-50 \%)$, combined with false positive rates from 15 to $30 \%$, again with the exception of PON:POP.

Figure 4 demonstrates the benefit of 2D models for the predictive capacity of especially nutrient balance ratios and PON:POP, but the latter remained anyhow a very modest predictor for $\mathrm{N}$ and P limitation, and showed 50:50 true and false positive rates for combined limitation. TN:TP ratio was the least reliable predictor after PON:POP for all limitation categories. DIN:TP and TNB scored the highest true positive and similar false positive rates for both $\mathrm{N}$ and $\mathrm{P}$ limitation, followed closely by DIN:DIP and BAN:BAP. The same overall ranking applied to prediction of combined limitation, except for lowered true positive rate of DIN:TP, although the modest true positive rates for combined limitation predictions were more widely scattered than for the single limitation categories.

\section{Threshold N:P Ratios}

Figure 5 shows estimated threshold N:P ratios with $95 \%$ confidence limits for all the indicators that have the Redfield N:P ratio as nominal value. We 


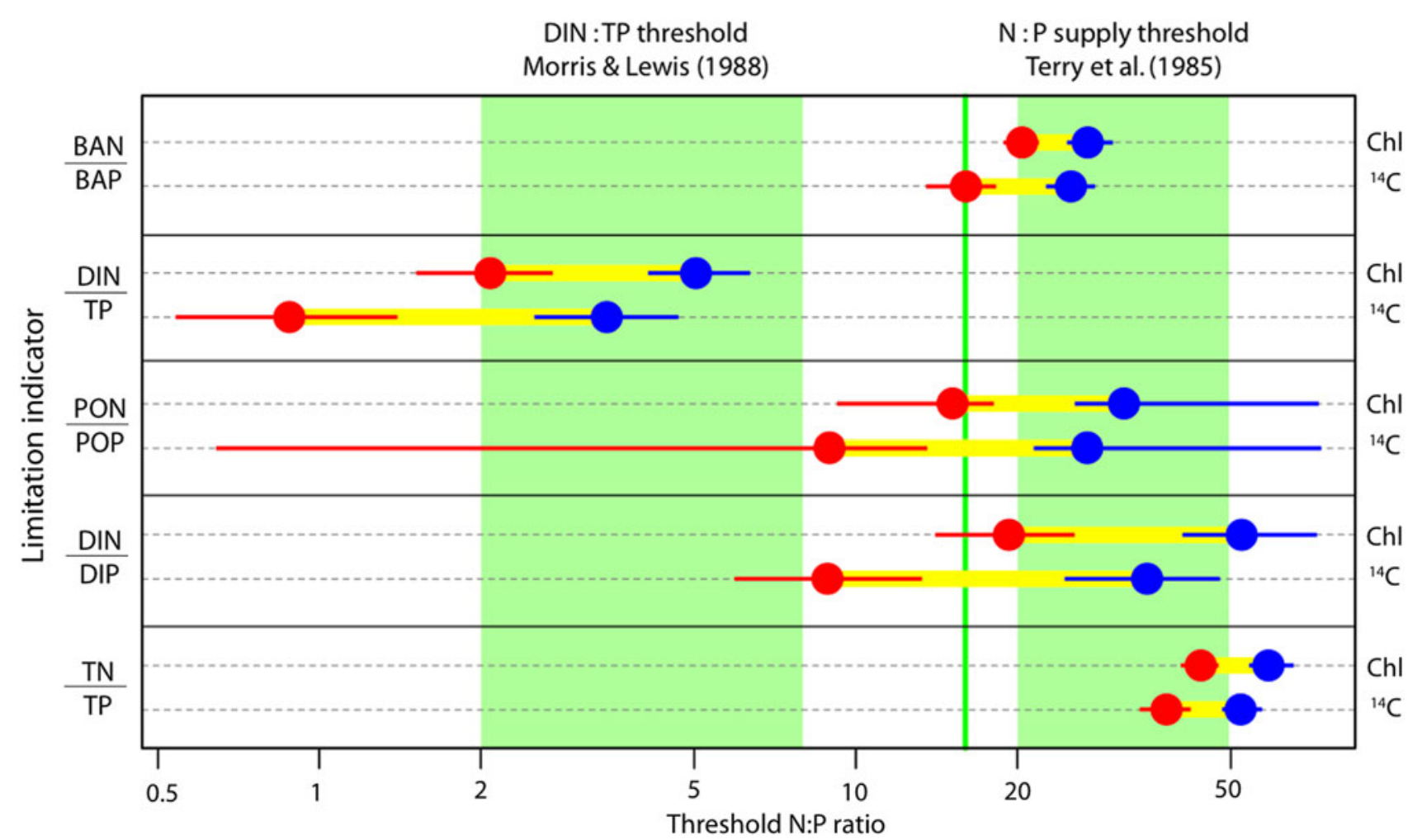

Figure 5. Thresholds for the transitions from $\mathrm{N}$ to $\mathrm{N}+\mathrm{P}$ limitation (red symbols) and $\mathrm{N}+\mathrm{P}$ to $\mathrm{P}$ limitation (blue symbols) according to equation (3). Only the five indicators with the Redfield ratio (16:1 by atoms; indicated by the green vertical line) as nominal value are shown. Shaded green areas correspond to published N:P thresholds for bioassays using the DIN:TP indicator (Morris and Lewis 1988), and for the N:P supply ratio in continuous cultures (Terry and others 1985). For each indicator, the upper part shows thresholds estimated using chlorophyll $a$ as response parameter, while the lower part shows the corresponding thresholds from the ${ }^{14} \mathrm{C}$ uptake response. Error bars are $95 \%$ confidence limits based on 1000 bootstrap samples from the original data set.

have chosen to exclude the nutrient balance indicators (TNB and PNB) which were the ones that showed the strongest departures from concentration-independent threshold ratios (that is, where the 2D models (equation (2)) gave highest improvement in predictive power, as judged from the ROC plots (Figure 4)), and which also do not have the Redfield ratio as a natural reference point.

The DIN:TP indicator thresholds were far below the others, and also significantly below the Redfield ratio, but not significantly different from those estimated from earlier studies (Morris and Lewis 1988), at least not when using the chlorophyll a response based predictor. PON:POP had thresholds closest to the Redfield ratio, although the low predictive power of this indicator is also reflected by the width of the confidence limits. The other indicators shown in Figure 5 generally had thresholds on the upper side of the Redfield ratio, and are more in line with threshold $\mathrm{N}: \mathrm{P}$ supply ratios from continuous culture experiments (Terry and others 1985), with most of the threshold ratios for the transitions to pure P limitation being significant higher than Redfield. Figure 5 also shows a strikingly consistent tendency for threshold $\mathrm{N}: \mathrm{P}$ ratios based on data using chlorophyll $a$ response parameter to be higher than corresponding values based on ${ }^{14} \mathrm{C}$ uptake as response parameter.

\section{Discussion}

\section{Ecological Interpretation and Predictive Power of Indicators}

Different nutrient fractions represent either nutrient supplies for planktonic organisms, cellular composition of the organisms, or combinations of both. The original concept of Redfield $(1934,1958)$ was built on large-scale stoichiometric convergence of phytoplankton biomass and their dissolved nutrient supply, through homeostatic regulation of the Ocean via phytoplankton metabolism. At global and evolutionary scales, comparisons of nutrient ratios of the 'supply side' and 'biomass side' should 
therefore yield information on transitory imbalances, and thus on nutrient limitation experienced by plankton.

It is less than obvious whether this concept is applicable at the decadal time scales of significant changes in anthropogenic nutrient supply to spatially confined or temporally dynamic water bodies, be it lakes or coastal areas, despite the extensive application of the Redfield ratio within ecology and management. However, in spite of repeated requests for reliable nutrient limitation indicators (for example, Howarth and Marino 2006), we know only about one study which has addressed the problem in a similar heuristic and systematic manner as done here. In a study performed in Colorado mountain lakes, Morris and Lewis (1988) compared a partly similar set of limitation indicators and came up with a closely matching ranking among them.

Although nutrient limitation has traditionally referred to autotrophic plankton, it has become increasingly evident that stoichiometric interactions within natural planktonic food webs, composed of autotrophic, mixotrophic and heterotrophic multispecies compartments, directly influence all nutrient fractions. Nutrient fractions are further affected by detrital compartments of varying biological availability. Depending on the choice of nutrient fractions for limitation assessments, the ecological interpretation of indicators will thus vary, and the same could be expected for the abilities of the indicators to predict in situ nutrient limitation of ambient phytoplankton.

Our 'standard' N:P ratios represent a supply ratio (DIN:DIP), a biomass proxy ratio (PON:POP), and total nutrient pools (TN:TP). There were large differences among these indicators, both in their ability to predict the limitation pattern (Figures 2, 3 , and 4, Table 1), and in their numeric thresholds for limitation shifts (Table 1; Figure 5).

The worst performing indicators were TN:TP and PON:POP, strongly indicating that the pools of non-autotrophic and/or biologically unavailable nutrient fractions can effectively mask the nutrient status of autotrophs. The wide divergence between the thresholds for TN:TP (very high) and the PON:POP (close to Redfield) ratios reflects the existence of a large pool of dissolved nitrogen unavailable for primary production. This is in line with the presence of a considerable pool of dissolved organic nitrogen in the study area (Supplementary material). Only a minor share (10-20\%) of this DON pool is degradable by bacterioplankton over a period of a week (Lignell and others 2008). Thus, ratios of total nitrogen and phosphorus are clearly misleading when it comes to predicting the in situ nutrient limitation of autotrophs. This is obviously aggravated in the Baltic Sea and other coastal systems strongly affected by their catchment. In spite of overall high TN:TP ratios (atomic TN:TP ratio $52 \pm 19.2$, mean \pm 1 SD), phytoplankton communities exhibited $\mathrm{N}$ and $\mathrm{P}$ limitation at similar frequencies (Tamminen and Andersen 2007).

The supply side 'standard' indicator, DIN:DIP, performed generally well, but it yielded limitation information departing from the traditional usages of Redfield ratio in two respects: the thresholds for changes between $\mathrm{N}, \mathrm{N}+\mathrm{P}$, and $\mathrm{P}$ limitation exceeded clearly Redfield N:P (Table 1), and the minor but significant concentration-dependency (thresholds decreasing with increasing amounts of dissolved nutrients) indicates that the requirement for DIP increases over-proportionally with increasing dissolved nutrients. However, the concentration dependency in Figure 3B should not be mistaken as eutrophication gradient. In fact, the most eutrophicated sites included in this study were characterized by overall low concentrations of dissolved inorganic nutrients and exhibited N-limitation (Tamminen and Andersen 2007).

With the exception of the DIN:TP ratio, the performance of the more elaborate 'composite' and 'balance' ratios did not appear to substantiate their potential theoretical benefits. It remains open whether the slim gains of increased complexity are due to cumulative measurement uncertainties of several nutrient fractions, or whether their underlying assumptions on biological availability, or on balance between supply and demand, are not sufficiently realistic.

Only the balance type indicators (TNB and PNB) seem to have substantial performance gains when going from $1 \mathrm{D}$ to $2 \mathrm{D}$ models, as judged by the lengths of the corresponding tails in Figure 4. Assuming that organisms with high N:P biomass ratios require corresponding high N:P supply (and vice versa), we expected these ratios to follow unity. This assumption was clearly falsified by co-limitation showing a negative relationship between dissolved and total (or particulate) fractions (Figure 4B, D): high TN:TP or PON:POP ratios generally correspond to $\mathrm{P}$ limitation which is made more severe under conditions of simultaneously high DIN:DIP ratios, and vice versa.

The best performing indicators, DIN:DIP, DIN:TP, and BAN:BAP, show rather little performance gain from the more complex 2D models, suggesting that all of these can successfully be used for deriving concentration-independent (ID) threshold ratios for $\mathrm{N}$ vs. P limitation. 


\section{Predicting N Limitation vs. Predicting P Limitation}

The threshold ratios for DIN:DIP and BAN:BAP are relatively near the Redfield ratio for the transition from N-limitation to combined limitation, while the transitions to pure P-limitation are in all cases significantly above Redfield (Figure 5). The ranges spanned by these two indicators fit reasonably well with observed ranges of critical N:P supply ratios in phytoplankton cultures (for example, Terry and others 1985; Leonardos and Geider 2004). Although threshold N:P supply ratios are known to differ among species and also to be strongly influenced by growth conditions (Leonardos and Geider 2004), it seems reasonable to interpret both the DIN:DIP and BAN:BAP concentration ratios as proxies for the actual N:P supply ratios experienced by the initial plankton communities in our experiments.

On the other hand, DIN:TP, which was the overall best performing indicator both in our study (Table 1) and in Morris and Lewis (1988), gave threshold ratios almost an order of magnitude lower than DIN:DIP (Figure 5). Since it seems impossible for DIN:TP and DIN:DIP to both reflect the N:P supply ratio at the same time, the key to the relative success of DIN:TP must be that it measures relative $\mathrm{N}: \mathrm{P}$ availability on a time scale more relevant to phytoplankton growth.

Morris and Lewis (1988) argued that DIN is the best predictor for available nitrogen because luxury uptake of $\mathrm{N}$ is negligible, while high capacity for luxury $\mathrm{P}$ uptake and fast turnover of dissolved $\mathrm{P}$ makes TP a better predictor of available phosphorus. Total $\mathrm{P}$ pool also includes organic $\mathrm{P}$, which is known to become available for phytoplankton through induction of alkaline phosphatase activity at times of low inorganic $P$ availability (Tanaka and others 2006). The fact that Morris and Lewis (1988) found critical DIN:TP ratios in the same range as our study (2-8 mol:mol), despite study objects as different as Colorado mountain lakes and Baltic Sea coastal areas, suggests that this indicator might have validity across considerable ranges of salinity and eutrophication.

\section{Combined Limitation}

Our results support the meta-analysis of Elser and others (2007) showing that combined $\mathrm{N}$ and $\mathrm{P}$ limitation is more common than previously thought. At the same time, our models have lower success rate in predicting combined limitation than the two other categories. This difficulty may be related both to the heterogeneous nature of combined limitation, and also to technical issues with the one-dimensional limitation indicator we have used in this analysis.

Our combined limitation class is the result of pooling the primary and exclusive combined limitation classes identified by Tamminen and Andersen (2007), which are probably more conceptually different than their counterparts for $\mathrm{N}$ or P limitation. The first would correspond to what Arrigo (2005) calls community co-limitation, where different populations in the community are limited by different nutrients, while the second is probably more a case of biochemical co-limitation (Arrigo 2005), where a single dominant population is limited by both nutrients simultaneously.

Moreover, the inherent information loss in compressing the original three-dimensional limitation probability vector into a one-dimensional limitation index is probably highest in the central region of the index. Consider for example the single red dot close to the center of Figure 1, which was an experiment with such weak and noisy responses that small changes in the input data gave significant probabilities for all 3 limitation classes, but with the $\mathrm{N}$ limitation probability slightly higher than the others. The higher false positive rate for $\mathrm{N}$ limitation than for P limitation (Figure 4) is mainly caused by the inability to separate $\mathrm{N}$ limitation from combined limitation. This is perhaps best illustrated by the 2D models (for example, DIN:TP; Figure 3D), where the blue P limitation dots clearly separate out while the red $\mathrm{N}$ limitation dots remain intermingled with yellow combined limitation dots.

\section{Seston vs. Phytoplankton Ratios: Constraining Mechanisms in Natural Assemblages}

The poor ability of the particulate $\mathrm{N}: \mathrm{P}$ ratio (PON:POP) to predict nutrient limitation is reflected in the relative constancy of seston composition, despite wide variability in dissolved and total nutrient fractions. Experimental studies on the relationship between $\mathrm{N}: \mathrm{P}$ supply ratio and cell composition are limited and partly conflicting: Rhee (1978) found that cell N:P of Scenedesmus cultures closely matched the N:P of the supply, while Goldman and others (1979) found cell N:P in Dunaliella cultures to converge to the Redfield ratio over a range of $\mathrm{N}: \mathrm{P}$ supply ratios.

Klausmeier and others (2004) used a mathematical model to show that these contrasting results may be reconciled by considering differences 
in growth conditions: constant cell N:P may be sustained over a range of N:P supply at high growth rate, as in Goldman and others (1979), while it will to a larger extent reflect the supply $\mathrm{N}: \mathrm{P}$ at low growth rate, as in Rhee (1978). Nutrient depleted cells have higher internal N:P ratios than nutrient replete cells, the latter requiring relatively more $\mathrm{P}$ to $\mathrm{N}$ to maintain fast cellular growth (Geider and LaRoche 2002). The analysis underlying the growth responses in the current study is sensitive to relative stimulation of growth. Even relatively replete cells may still respond to addition of $\mathrm{N}$ or $\mathrm{P}$ if other factors support higher growth. In accordance to this, the threshold DIN:DIP ratio decreases with increasing amount of nutrients (Figure 3B).

The results indicate that the critical $\mathrm{N}: \mathrm{P}$ supply ratio for stimulating phytoplankton growth is not constant, but decreases with increasing growth rate, reflecting systematic changes in cellular composition between nutrient deplete and replete cells. Klausmeier and others (2004) conclude that our understanding of the physiological regulation of $\mathrm{N}$ and $\mathrm{P}$ uptake is limited, and that the current generation of Liebig-type models of co-limitation is probably too simplistic.

Our findings match those of Hall and others (2005), who demonstrated low seston N:P variability on widely variable $\mathrm{N}$ :P supply ratios in both natural and artificial freshwater systems (ponds and mesocosms), and also supported their findings by a survey of literature data from an even wider range of systems (lentic and lotic; marine, freshwater, and terrestrial).

It should be kept in mind that seston $\mathrm{N}: \mathrm{P}$ will in any case be a blunted signal for the cellular composition of the phytoplankton, since the material captured on a filter will be a complex mixture of autotrophic, heterotrophic, and detrital constituents. Thus, low variability of seston N:P need not be in conflict with variable phytoplankton stoichiometry, if the phytoplankton of the initial community constitutes a minor component partly masked by nutrient-poor detritus and more homeostatic heterotrophs like metazoan grazers.

\section{Concluding Remarks}

Our analysis shows that standard monitoring parameters like DIN, DIP, and TP may be used to predict in situ phytoplankton $P$ limitation with high confidence and high power $(80 \%$ true positive rate, $5 \%$ false positive rate), while the discrimination between combined $(\mathrm{N}+\mathrm{P})$ limitation and single limitation by $\mathrm{N}$ is more prone to false positives.
From the management point of view, it is important to be aware that prevailing $\mathrm{N}$-limitation will be more difficult to detect from nutrient data than prevailing P-limitation.

DIN:TP performed the best in predicting prevailing limitation, and the ID model yielded numeric thresholds between limitation categories. There are also other aspects supporting its use in inferring nutrient limitation from typical monitoring data. While DIN:DIP and BAN:BAP showed relatively similar abilities, many existing data sets do not include particulate nutrients (PON and POP), which makes the calculation of BAN:BAP impossible. As DIP measurements are expected to have higher measurement noise than TP, especially for DIP concentrations close to analytical detection limits, one would also expect DIN:DIP to be a more noisy indicator than DIN:TP.

Besides the apparent heuristic value of our results, some more general findings are intriguing but cannot be given a satisfactory explanation by the current analysis. The novel observation that all indicators showed lower threshold N:P ratios when using photosynthetic ${ }^{14} \mathrm{C}$-uptake as response parameter, than when using chlorophyll a (Figure 5), is too obvious and consistent to be overlooked. While our experiments were not designed to discuss this phenomenon in detail, we want to point out that chlorophyll $a$ is a more integrative indicator than a short-term rate measurement such as 14C fixation, which is more affected by light availability, temperature and physiological state of the phytoplankton.

The widespread and strong positive synergistic effects of $\mathrm{N}$ and $\mathrm{P}$ enrichment (Elser and others 2007) have obvious consequences for management of nutrient discharges (Conley and others 2009; Paerl 2009). N and P play unequal roles in phytoplankton nutrient uptake and growth. Our results clearly indicate that standard 'Redfield conversions' between major nutrients have limited applicability in phytoplankton ecology. More adequate descriptions of multiple limitation are needed also for planktonic food web studies, as well as for modeling eutrophication dynamics in coastal systems.

\section{ACKNOWLEDGMENTS}

The data originate from a Finnish project PELAG III (for full acknowledgments, see Tamminen and Andersen 2007). Aspects of the in-depth data analysis have been funded by the EU 5th Framework Project DANLIM (EVK3-CT-2001-00049, coordinator Risto Lignell) and the 6th Framework Project THRESHOLDS (GLOBAL/IP/02/0257, coordinator 
Carlos Duarte). Comments by two anonymous reviewers are acknowledged.

\section{OPEN ACCESS}

This article is distributed under the terms of the Creative Commons Attribution Noncommercial License which permits any noncommercial use, distribution, and reproduction in any medium, provided the original author(s) and source are credited.

\section{REFERENCES}

Andersen T, Saloranta TM, Tamminen T. 2007. A statistical procedure for unsupervised classification of nutrient limitation bioassay experiments with natural phytoplankton communities. Limnol Oceanogr Methods 5:111-18.

Arrigo KR. 2005. Marine microorganisms and global nutrient cycles. Nature 437:349-55.

Cloern JE. 2001. Our evolving conceptual model of the coastal eutrophication problem. Mar Ecol Prog Ser 211:223-53.

Conley DJ, Paerl HW, Howarth RW, Boesch DF, Seitzinger SP, Havens KE, Lancelot C, Likens GE. 2009. Controlling eutrophication: nitrogen and phosphorus. Science 323:1014-15.

Elser JJ et al. 2007. Global analysis of nitrogen and phosphorus limitation of primary producers in freshwater, marine and terrestrial ecosystems. Ecol Lett 10:1135-42.

Falkowski PG. 2000. Rationalizing elemental ratios in unicellular algae. J Phycol 36:3-6.

Fawcett T. 2006. An introduction to ROC analysis. Pattern Recogn Lett 27:861-74.

Geider RJ, La Roche J. 2002. Redfield revisited: variability of $\mathrm{C}: \mathrm{N}: \mathrm{P}$ in marine microalgae and its biochemical basis. Eur $\mathrm{J}$ Phycol 37:1-17.

Goldman JC, McCarthy JJ, Peavey DG. 1979. Growth rate influence on the chemical composition of phytoplankton in oceanic waters. Nature 279:210-15.

Grasshoff K, Ehrhardt M, Kremling K. 1983. Methods of seawater analysis. Weinheim, Germany: Verlag Chemie.

Hall SR, Smith VH, Lytle DA, Leibold MA. 2005. Constraints on primary producer $\mathrm{N}: \mathrm{P}$ stoichiometry along $\mathrm{N}: \mathrm{P}$ supply ratio gradients. Ecology 86:1894-904.

Howarth RW, Marino R. 2006. Nitrogen as the limiting nutrient for eutrophication in coastal marine ecosystems: evolving views over three decades. Limnol Oceanogr 51:364-76.

Johnson JB, Omland KS. 2004. Model selection in ecology and evolution. Trends Ecol Evol 19:101-8.

Klausmeier CA, Litchman E, Levin SA. 2004. Phytoplankton growth and stoichiometry under multiple nutrient limitation. Limnol Oceanogr 49:1463-70.

Leonardos N, Geider RJ. 2004. Responses of elemental and biochemical composition of Chaetoceros muelleri to growth under varying light and nitrate:phosphate supply ratios and their influence on critical N: P. Limnol Oceanogr 49:2105-14.

Lignell R, Hoikkala L, Lahtinen T. 2008. Effects of inorganic nutrients, glucose and solar radiation on bacterial growth and exploitation of dissolved organic carbon and nitrogen in the northern Baltic Sea. Aquat Microb Ecol 51:209-21.

Morris DP, Lewis WM. 1988. Phytoplankton nutrient limitation in Colorado mountain lakes. Freshw Biol 20:315-27.

Paerl HW. 2009. Controlling eutrophication along the freshwater-marine continuum: dual nutrient $(\mathrm{N}$ and $\mathrm{P})$ reductions are essential. Estuaries Coasts 32:593-601.

Ptacnik R, Jenerette GD, Verschoor AM, Huberty AF, Solimini AG, Brookes JD. 2005. Applications of ecological stoichiometry for sustainable acquisition of ecosystem services. Oikos 109:52-62.

R Development Core Team. 2005. R: a language and environment for statistical computing. R Foundation for Statistical Computing, Vienna, Austria (http://www.R-project.org).

Redfield AC. 1934. On the proportions of organic derivatives in sea water and their relation to the composition of plankton. Liverpool: James Johnstone Memorial Volume. pp 176-92.

Redfield AC. 1958. The biological control of chemical factors in the environment. Am Sci 46:205-21.

Reynolds CS, Davies PS. 2001. Sources and bioavailability of phosphorus fractions in freshwaters: a British perspective. Biol Rev 76:27-64.

Rhee GY. 1978. The effect of N:P atomic ratios and nitrate limitation on algal growth, cell composition, and nitrate uptake. Limnol Oceanogr 23:10-25.

Schindler DW. 1977. Evolution of phosphorus limitation in lakes. Science 195:260-2.

Solorzano L, Sharp JH. 1980. Determination of total dissolved phosphorus and particulate phosphorus in natural waters. Limnol Oceanogr 25:754-8.

Tamminen T. 1982. Effects of ammonium effluents on planktonic primary production and decomposition in a coastal brackish water environment. I. Nutrient balance of the water body and effluent tests. Neth J Sea Res 16:455-64.

Tamminen T, Andersen T. 2007. Seasonal phytoplankton nutrient limitation patterns as revealed by bioassays over Baltic Sea gradients of salinity and eutrophication. Mar Ecol Prog Ser 340:121-38.

Tanaka T, Henriksen P, Lignell R, Olli K, Seppälä J, Tamminen T, Thingstad TF. 2006. Specific affinity for phosphate uptake and specific alkaline phosphatase activity as diagnostic tools for detecting phosphorus-limited phytoplankton and bacteria. Estuaries Coasts 29:1226-41.

Terry KL, Laws EA, Burns DJ. 1985. Growth rate variation in the N:P requirement ratio of phytoplankton. J Phycol 21:323-9.

Vitousek P, Howarth R. 1991. Nitrogen limitation on land and in the sea: how can it occur? Biogeochemistry 13:87-115. 